\title{
Are cognitive changes the first symptoms of Huntington's disease? A study of gene carriers
}

\author{
V Hahn-Barma, B Deweer, A Dürr, C Dodé, J Feingold, B Pillon, Y Agid, A Brice, \\ B Dubois
}

\begin{abstract}
Background-Huntington's disease is a neurodegenerative disorder due to an excessive number of CAG repeats in the IT15 gene on chromosome 4. The first symptoms are typically choreic movements or psychiatric disorders, whereas global cognitive decline generally becomes obvious later. This study was aimed at detecting early subtle cognitive deficits in asymptomatic gene carriers.

Methods-As part of the testing procedure for predictive diagnosis of Huntington's disease, 91 asymptomatic at risk candidates had a neuropsychological examination, evaluating global efficiency, attention, memory (Wechsler memory scale and California verbal learning test), and executive functions.
\end{abstract}

Results-The groups of carriers $(n=42)$ and non-carriers $(n=49)$ differed only on a few memory variables. When we considered the group of gene carriers as a whole, significant correlations emerged between the number of CAG repeats and (a) performance on several tests of executive functions, and $(b)$ performance on the hard pairs associates of the Wechsler memory scale. Further analysis of performance on this memory subtest led to the division of the group of carriers into two subgroups, without any overlap. The performance of subjects without cognitive deficits $(n=32)$ was similar to that of noncarriers on all tests. The subjects with cognitive deficits $(n=10)$ differed from both carriers without cognitive deficits and non-carriers over a wide array of variables measuring executive functions and memory. Moreover, qualitative aspects of the performance of carriers with cognitive deficits in the California verbal learning test closely resembled those of patients diagnosed as having Huntington's disease.

Conclusion-This suggests that these subjects already have Huntington's disease, despite a total lack of motor and psychiatric signs. An ongoing follow up study is testing the prediction that they will develop the full range of symptoms of the disease earlier than carriers without cognitive deficits.

\section{(F Neurol Neurosurg Psychiatry 1998;64:172-177)}

Keywords: Huntington's disease; asymptomatic gene carriers; cognitive impairment
Huntington's disease is an autosomal dominant disorder with complete lifetime penetrance, characterised by insidious onset of symptoms associating choreic movements, affective disorders, and cognitive impairment. ${ }^{1}$ The diagnosis is typically based on the appearance of the first motor symptoms, and confirmed by the presence of an excessive number (over 36) of CAG repeats in the IT15 gene on chromosome $4 .^{2}$ Together with genetic analysis, neuropsychological testing has been performed either on asymptomatic subjects at high risk for Huntington's disease (indirect method $)^{3-5}$ or asymptomatic gene carriers (direct method) ${ }^{6-8}$ Some of these studies with neuropsychological evaluation have reported that gene carriers were less efficient than noncarriers in the domains of executive functions and, to a lesser degree, memory. However, the numbers of asymptomatic patients were rather small in most studies and the differences were neither robust nor consistently found.

One explanation for these inconsistent results may be that, in these studies, the groups of gene carriers were considered as a whole, including variable proportions of both at risk subjects with no cognitive impairment (presumably far from developing the disease) and at risk subjects with subtle cognitive impairment. To avoid this confounding effect, we performed a three step analysis of the performance of gene carriers, (1) by comparing gene carriers and non-carriers on a large neuropsychological battery, (2) by looking for correlations between the number of CAG repeats and efficiency on the neuropsychological tests within the group of gene carriers, and (3) by subdividing the group of gene carriers into two subgroups on the basis of their performance on the memory subtest best correlated with CAG repeats. The underlying hypothesis was that gene carriers with cognitive impairment already have Huntington's disease, despite total lack of motor abnormalities, and will develop the full range of the symptoms earlier than normally efficient gene carriers. If this hypothesis is valid, it should be possible to predict the onset of the disease in the absence of any motor or affective disorder, on the basis of some early modifications of cognitive efficiency which might constitute sensitive markers.

Methods and subjects INCLUSION PROCEDURE

During a period of three years, 99 candidates at the Salpêtrière Hospital, Paris, France, followed the presymptomatic diagnosis procedure until the genetic result. Of these, seven were 
excluded from this study on the basis of possibly confounding factors: concomitant neurological disorders (two), depression (two), advanced age $>65$ (two), education level $<$ six years (one). Another subject was excluded after the genetic analysis because he was in the so-called intermediate zone ( $31 \mathrm{CAG}$ repeats). Thus, 91 subjects ( 32 men and 59 women) were included in the study; their mean age was 33.3 (SD 8.1) (range 18-53) years; their mean level of education was 12.9 (SD 3.2) (range 7-17) years.

The standard procedure for predictive testing of Huntington's disease was run by a multidisciplinary team and included genetic counselling, neurological examination by a neurologist specialised in Huntington's disease, psychological interviews, social evaluation, and neuropsychological testing. This double blind clinical assessment lasted about three months before the molecular analysis was undertaken.

This procedure takes into account the recommendations of the committee of the International Huntington Association (IHA) and the World Federation of Neurology (WFN). ${ }^{9}$ All candidates included in the procedure for predictive diagnosis of Huntington's disease were: (1) at risk - that is, with a family history of identified Huntington's disease cases (see below); (2) asymptomatic - that is, free of any motor or psychiatric symptoms characteristic of Huntington's disease; (3) “consenting and informed"- that is, understanding and accepting the test by written consent.

CLINICAL ASSESSMENT

An exhaustive interview carried out by the geneticist and the neurologist verified the positive family history of Huntington's disease; the clinical diagnosis of Huntington's disease in affected family members established the existence of an a priori risk in the candidate according to the genealogy. All candidates were at a $50 \%$ risk, except one who was at $25 \%$, because his at risk mother was still unaffected at 42 years. Molecular confirmation for at least one affected relative was obtained in $90 \%$ (82 of 91) of subjects. In the remainder, the clinical diagnosis in the affected relatives was strongly suggestive of Huntington's disease. In addition to a complete neurological evaluation, the presence of abnormal movements was especially looked for. There were no subjects with even minor signs of chorea, brisk reflexes in the lower limbs, oculomotor disturbances, motor impersistence, parkinsonism, or tremor, except for one subject with physiological enhanced postural tremor. Lastly, no subjects presented psychiatric disorders at the time of examination.

\section{MOLECULAR ANALYSIS}

Blood samples were taken from all subjects to determine the number of CAG repeats as previously reported. ${ }^{10}$ Of the 91 subjects, 49 $(53.8 \%)$ were non-carriers (less than 30 CAG repeats in both alleles) and $42(46.2 \%)$ were gene carriers (more than 36 CAG repeats in one allele of the IT15 gene).
NEUROPSYCHOLOGICAL TESTING

The neuropsychological battery used included various tests known to be sensitive to Huntington's disease, ${ }^{11-14}$ evaluating global cognitive efficiency, attentional capacities, executive functions, and memory performance. Cognitive efficiency was assessed with the mini mental state examination (MMSE) ${ }^{15}$ and the Mattis dementia rating scale. ${ }^{16}$ Attentional capacities were assessed by the digit span subtest of the Wechsler adult intelligence scale revised, ${ }^{17}$ and the Stroop test. ${ }^{18}$ In a previous study, ${ }^{19}$ we showed that in patients with choreic movements, this battery correctly discriminates between those with sporadic Huntington's disease and those with non-Huntington's disease chorea.

Tests of executive functions included a score of lexical fluency, corresponding to the sum of the words beginning with "P", " $R$ ", and " $V$ " in two minutes divided by three, ${ }^{20}$ the trail making test, ${ }^{21}$ with scores transformed into base 10 logarithms, and the digit symbol and arithmetic subtests of the Wechsler adult intelligence scale revised (WAIS-R).

Memory efficiency was evaluated with the Wechsler memory scale (WMS) ${ }^{22}$ with delayed recall for logical memory, visual retention, and paired associates, and the California verbal learning test (CVLT), ${ }^{23}$ which allows both quantitative and qualitative assessment of memory performance. This last test includes the following steps: (1) learning, in five trials, of a 16 item shopping list (Monday list) belonging to four embedded semantic categories; (2) acquisition in one trial of an interference list of 16 shopping items (Tuesday list) belonging to four embedded semantic categories, of which two are shared with the Monday list; (3) short delay free recall of the Monday list; (4) short delay cued recall of the Monday list, providing the subject with each of the four category names to facilitate recall; (5) 20 minute delayed free recall of the Monday list; (6) delayed cued recall of the Monday list; (7) recognition of the Monday list items from various foils, including interference list words that are semantically related or unrelated to target words; novel words that are prototypical of the semantic categories used in the Monday list; novel words phonetically similar to target words, and novel words that are semantically and phonetically unrelated to target words. Besides the recall and recognition subtest scores, the analysis of performance included evaluation of perseverations (multiple productions of the same item within the same trial), intrusions (production of extra list words), consistency of recall from trial to trial, semantic clustering, and serial clustering during learning of the Monday list, ${ }^{23}$ as well as false alarms and discriminability at recognition (a non-parametric index of accuracy of recognition, taking into account both misses and false positives). ${ }^{24}$

The mood state of all subjects was assessed with the Montgomery and Asberg depression rating scale ${ }^{25}$ and the gravity of anxiety scale of Covi. ${ }^{26}$ 
STATISTICAL ANALYSIS

Statistical comparisons used parametric (analysis of variance (ANOVA), unpaired Student's $t$ test) or non-parametric (KruskalWallis test, Mann-Whitney $U$ test) tests, as appropriate. Given the large number of comparisons, the significance level was set at 0.01 .

\section{Results}

COMPARISON OF NON-CARRIERS AND GENE CARRIERS

These two groups did not differ in age (33.2 (SD 8.8) for non-carriers and 33. 4 (SD 7.4) for carriers), number of years of education (non-carriers $=12.8$ (SD 3.2); carriers $=13.2$ (SD 3.0)); and $\mathrm{M} / \mathrm{F}$ sex ratio (non-carriers $=17$ / 32; carriers $=15 / 27$ ). They were also similar on depression (5.3 (SD 3.3) and 5.3 (SD 4.2)) and anxiety (3.3 (SD 1.6) and 3.8 (SD 2.1)) scores.

Global efficiency was similarly preserved in both groups (MMSE mean scores>29.5; Mattis DRS mean scores>141). For attentional capacities, the performance was similar and in the normal range in both groups, although direct and reverse digit spans were lower than expected (direct $=6.5$; reverse $=4.7$ ), given the age and level of education of the subjects. Executive function scores yielded only a tendency for lower performance in gene carriers on the arithmetic subtest of the WAIS-R (9.4 in gene carriers $v 10.7$ in non-carriers; NS).

The difference between groups was significant on delayed recall of the logical memory subtest (13.4 (SD 3.1) and 11.4 (SD 3.4); $t$ $(89)=2.86 ; \mathrm{p}<0.006)$, as well as on immediate recall of hard paired associates (10.9 (SD 0.8) and $9.8($ SD 2.4); $\mathrm{t}(89)=2.79 ; \mathrm{p}<0.007)$ of the WMS. The groups were similar on the visual retention subtest. At the CVLT, significant differences emerged for recognition (15.8 (SD $0.4)$ and 15.3 (SD 1.0) hits; $z=2.98 ; p<0.003)$ and recognition discriminability (99.1 (SD 1.9) and 97.0 (SD 3.9); $z=2.86 ; p<0.005$ ).

For some variables, variance was greater in the group of gene carriers than in the group of non-carriers. This is suggestive of heterogeneity of the group of gene carriers, which would comprise both at risk subjects without significant impairment and subjects with cognitive abnormalities. Is such heterogeneity related to the variability of the CAG repeats at an individual level?

NUMBER OF CAG REPEATS AND

NEUROPSYCHOLOGICAL PERFORMANCE IN GENE CARRIERS

Among the group of gene carriers, the number of CAG repeats in the IT15 gene on the chromosome 4 varied from 37 to 49 . We used linear regression to determine whether the number of repeats was correlated with scores on neuropsychological testing. The correlations proved to be significant for several tests aimed at assessing executive functions: Digit symbol subtest of the WAIS-R $(r=0.58 ; F$ $(1,40)=20.01 ; \mathrm{p}<0.0001) ;$ Stroop test, colours $(r=0.42 ; F(1,40)=8.42 ; \mathrm{p}=0.006)$; Stroop test, interference $(r=0.40 ; \mathrm{F}(1,40)=7.56$; $\mathrm{p}=0.009)$. For memory tests, the correlation was also highly significant for the paired associates subtest of the WMS $(r=0.47 ; F$ $(1,40)=11.31 ; \mathrm{p}=0.002)$; within this subtest, the performance on the hard pairs was correlated with the number of CAG repeats $(r=0.48 ; F(1,40)=11.78 ; \mathrm{p}=0.0014)$, whereas the performance on the easy pairs was not $(\mathrm{p}=0.35)$.

The correlations were close to the significance level (in each case $\mathrm{p}<0.04$ ) only for the Mattis DRS score, the trail making test (B-A), the Wechsler memory quotient, the number of "list A words" learned, and the consistency of recall at the CVLT.

COMPARISON OF NON-CARRIERS, "PAIRED ASSOCIATES IMPAIRED" GENE CARRIERS, AND “PAIRED ASSOCIATES UNIMPAIRED" GENE CARRIERS

The fact that the performance on the hard paired associates of the WMS was strongly correlated with the number of CAG repeats led us to further examine individual performance of gene carriers on this subtest. Ten out of the 42 subjects scored 8 or lower, whereas none of the 49 subjects in the group of non-carriers scored under 9. It was therefore possible operationally to define two different subgroups among gene carriers: a "paired associates impaired" group $(n=10)$ and a "paired associates unimpaired" group $(n=32)$.

The three groups did not differ in terms of sex ratio $M / F$ (non-carriers $17 / 32$; carriers, paired associates unimpaired group 10/22; carriers, paired associates impaired group 5/5); age (33.2 (SD 8.8) for non-carriers, 33.7 (SD 7.3) for carriers, paired associates unimpaired group, 32.6 (SD 7.9) for carriers, paired associates impaired group); years of education (non-carriers $=12.8$ (SD 3.2); carriers, paired associates unimpaired group $=13.7$ (SD 2.5); carriers, paired associates impaired group $=13.5$ (SD 3.9)), depression (noncarriers $=5.3$ (SD 3.3); carriers, paired associates unimpaired group $=5.9$ (SD 4.4), carriers, paired associates impaired group $=3.7$ (SD $3.2)$ ), and anxiety (non-carriers=3.3 (SD 1.5); carriers, paired associates unimpaired group $=3.7$ (SD 2.1); carriers, paired associates impaired group $=4.1$ (SD 2.1)) scores. They differed only in terms of number of CAG repeats on the expanded allele (noncarriers $<30$; carriers, paired associates unimpaired group $=42.3$ (SD 2.9), carriers, paired associates impaired group $=45.2$ (SD 2.4); $\mathrm{p}<0.01$ ).

Paired associates unimpaired carriers had a neuropsychological profile closely similar to that of non-carriers. By contrast, statistical comparisons between the two subgroups of gene carriers yielded a number of significant differences, which concerned global efficiency (Mattis DRS), executive functions (arithmetic and digit symbol subtests of the WAIS-R and verbal fluency), and memory (WMS and CVLT) (table). Paired associates impaired subjects performed significantly less well on verbal memory subtests of the WMS, and for initial learning of list A, free recall, cued recall, 
Table 1 Comparison of the three groups: non-carriers (NC), paired associates unimpaired (PAU), and impaired (PAI) carriers (mean (SD))

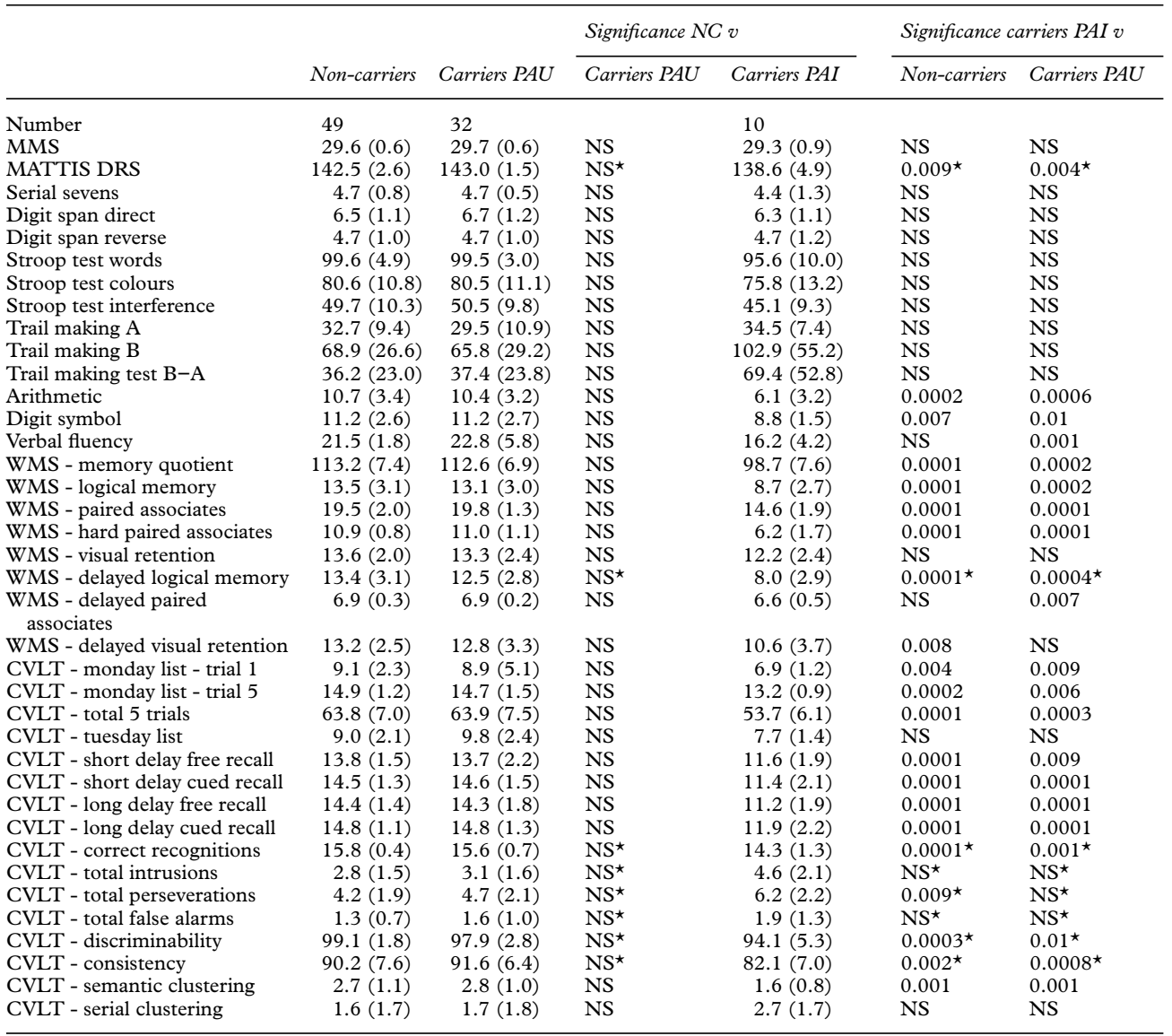

^ Mann-Whitney $U$ test ( $z$ test scores corrected for ties). Otherwise, unpaired Student's $t$ test. NS $=p>0.01$.

and number of hits at recognition of the CVLT. Further analysis of the CVLT disclosed that subjects in the paired associates impaired group were significantly less consistent from trial to trial during initial learning (they did not systematically retrieve the same items from trial to trial), and had a much less efficient semantic clustering strategy than subjects in the paired associates unimpaired group (the semantic clustering indices were 1.58 (SD 0.8) in the paired associates impaired group and 2.79 (SD 0.97) in the paired associates unimpaired group; $\mathrm{p}<0.001)$; lastly, subjects in the paired associates impaired group were also impaired at discriminating between target items and foils at recognition.

\section{NUMBER OF CAG REPEATS, COGNITIVE}

IMPAIRMENT, AND AGE AT ONSET

Despite nearly identical age at examination, the CAG repeat number was significantly higher in the cognitively impaired subgroup. According to the age at onset/CAG repeat number correlation for subjects in the same CAG repeat range (37-49), the age at onset of Huntington's disease for the cognitively impaired subgroup would seem to be four to five years earlier than for the cognitively unimpaired subgroup (correlation slope $=-1.7 ; r=0.312 ; \mathrm{p}<0.001 ;$ Dürr $e t$ al, unpublished data).

\section{Discussion}

Our study shows significant differences in cognitive performance of non-carriers and gene carriers for Huntington's disease. These differences concern verbal memory such as logical memory and paired associates subtests of the WMS, and numbers of hits and discriminability of recognition at the CVLT. By contrast, other authors have reported no sign of cognitive impairment, either in subjects at high risk for Huntington's disease or in gene carriers. ${ }^{72}{ }^{28}$ How might such discrepancies be accounted for? Differences in selected tests, on the one hand, and relatively low numbers of subjects in most studies, on the other hand, are certainly contributory factors. Another possibly relevant factor, stressed by Jossiassen $e t a l^{29}$ (but critically re-examined ${ }^{27}$ ), is that the variability of performance of subjects at high risk is greater than that of subjects at low risk.

Therefore, the second step of the analysis included a closer examination of the neuropsychological heterogeneity of the group of gene carriers. The correlational analyses indicated that, at least for tests of executive functions and the hard paired associates subtest of the WMS, such a heterogeneity is related to the number of CAG repeats. The fact that variability was highest for the paired associates subtest of the WMS, and particularly for the hard paired associates (for which the scores varied from 12 
(maximum score) to 2), is not surprising. Hard pairs are typically much more sensitive to brain damage than easy pairs, because they assess retention of new, unfamiliar associations (which requires the initiation of active research strategies in memory), as opposed to rather automatic recall of well learned verbal associations for the easy pairs. ${ }^{30}$ Moreover, in the study of Diamond et al, ${ }^{5}$ the paired associates subtest of the WMS was also the most sensitive in differentiating subjects with high and low risk for Huntington's disease. Studying parkinsonian patients with a new hard paired associates learning task, El Awar et $a l^{\beta 1}$ were also able to differentiate two subgroups, exhibiting low or high error scores.

In our view, the group of gene carriers actually comprises two subgroups, one of them already exhibiting cognitive impairmentdespite the absence of any motor or psychiatric disturbance - defined by a particular neuropsychological profile, characteristic of an early stage of the disease. Moreover, such a profile resembles that of patients with symptomatic Huntington's disease. The subgroup of cognitively unimpaired carriers was not different from subjects in the group of non-carriers in any way, and therefore must be considered as still normal. The subgroup of cognitively impaired carriers significantly differed from both the cognitively unimpaired subgroup and the non-carrier group on a large array of measures, including: most subtests of the WMS (with the notable exception of visual retention); most measures of the CVLT; some tests of executive functions (arithmetic and digit symbol subtests of the WAIS-R, verbal fluency); and global efficiency score (Mattis DRS). As stated earlier, the subgroups of gene carriers did not differ from each other in terms of depression or anxiety. Moreover, no subject in these subgroups exhibited any abnormal movement.

Rothlind et $a l^{28}$ also singled out and described a subject who, despite a normal neurological examination, produced a grossly abnormal performance on the Hopkins verbal learning test, whereas the other subjects at high risk were in the normal range. Two years later, she displayed eye movement abnormalities, motor impersistence, and mild choreiform movements, and the next year received a clinical diagnosis of Huntington's disease. According to Rothlind et al, this patient "appears to have been in the phase of disease that may be accompanied by subtle signs of cognitive abnormalities, but clearly is below threshold for diagnosis". This might well be also the case for the 10 gene carriers with cognitive impairment described here, as their neuropsychological profile presents several similarities with that of diagnosed patients with Huntington's disease ${ }^{13}$ : (1) these patients were impaired on the arithmetic and digit symbol subtests of the WAIS, which are generally performed poorly by patients with Huntington's disease ${ }^{11} 123233$; (2) the decrease of verbal fluency is also characteristic of patients with Huntington's disease $^{12}{ }^{34}$; (3) more generally, a dysexecutive syndrome has been found in demented patients with Huntington's disease $\mathrm{e}^{35}$ as well as in patients with early Huntington's disease ${ }^{36} ;(4)$ memory disturbance, found in this subgroup of subjects, is a prominent and early appearing cognitive feature of Huntington's disease. ${ }^{13}$

Data concerning the CVLT highlights strong quantitative as well as qualitative similarities between the subgroup of cognitively impaired subjects described here and diagnosed patients with Huntington's disease. ${ }^{37}$ Such similarities indicate that the CVLT is particularly suited for studying gene carriers or patients with Huntington's disease. In both studies, patients with Huntington's disease or cognitively impaired subjects exhibited deficits at learning the first list (first trial, last trial, and total) and on all free or cued recall trials, but were not more sensitive than normal controls (or, in this study, non-carriers and cognitively unimpaired subjects) to proactive interference (the free recall score for the second list was similar to that of the free recall score on the first trial of the first list). Interestingly, subjects in the cognitively impaired subgroup and patients with Huntington's disease also shared several qualitative features of memory performance: (1) during initial learning, they both displayed $(a)$ less consistency of recall from trial to trial; $(b)$ deficient use of a semantic clustering strategy, and (c) an accentuated recency effect; (2) subjects in the cognitively impaired group also tended to show more perseverations than subjects in cognitively unimpaired and non-carrier groups, whereas patients with Huntington's disease did have higher perseveration rates than controls in the study of Massman et $a l^{2}$; (3) at recognition, both groups (cognitively impaired subjects and patients with Huntington's disease) were slightly, but significantly, impaired at discriminating between targets and distractors (see table, discriminability index). Whereas this last aspect of performance may be indicative of mildly deficient encoding, impaired semantic clustering and inconsistent recall of words from trial to trial are illustrative of pronounced difficulties in initiating systematic retrieval strategies, reflecting an executive deficit. ${ }^{35} 363839$

These data indicate that it is possible to identify a cognitively impaired subgroup within "asymptomatic" gene carriers. Although these subjects are free of any neurological or psychiatric symptoms, they may well differ from the normal carriers in neuropathology; it may be that the longer repeat length leads to a less circumscribed neuropathology, or that the degenerative process is more advanced. Whether this subgroup will develop the disease earlier than cognitively unimpaired subjects remains to be determined in a follow up study. However, it can be anticipated that the presence of a significantly higher number of CAG repeats in the cognitively impaired gene carriers will be associated with an earlier mean age at onset in this subgroup (calculated to be at least four years), which would be strongly consistent with our overall results.

Our study establishes that cognitive changes without motor or psychiatric disturbances represent the first sign of Huntington's disease in a 
subset of gene carriers. The dysfunction of the caudate nucleus, evidenced by metabolic studies, ${ }^{40-42}$ can induce a dysexecutive syndrome and memory deficits even before the diagnosis of Huntington's disease is established. A follow up study of the gene carriers is in progress, and is designed to elucidate such questions, including the following: (1) what is the delay between the occurrence of subtle cognitive impairment and both the first motor symptoms and the diagnosis of Huntington's disease? (2) Will other subjects, currently in the cognitively unimpaired subgroup, also exhibit signs of cognitive impairment before motor or psychiatric disorders? (3) Will subjects in the cognitively impaired subgroup develop the full range of Huntington's disease symptoms before subjects in the cognitively unimpaired subgroup, as already suggested on the basis of the correlation between the number of CAG repeats and the age at onset?

Financial support was provided by ADRMGNP, CNAMTS and Financial support was provided by ADRMGNP, CNAMTS and Association Huntington France. We are grateful to Marcela Garguilo, Dr M Leboyer, and Bénédicte Prouvost for psychoso-
cial evaluation of the candidates and to Isabelle Lagroua and Corinne Zentar for technical assistance.

1 Caine ED, Fisher JM. Dementia in Huntington's disease. In Vinken PJ, Bruyn GW, Frederiks JA, eds. Neurobehavioral disorders, handbook of clinical neurology. Amsterdam: Elsevier, $1985 \cdot 305-10$.

2 Huntington's Disease Collaborative Research Group. A novel gene containing a trinucleotide repeat that is expanded and unstable on Huntington's disease chromosomes. Cell 1993;72:971-83.

3 Jason GW, Pajurkova EM, Suchowersky O, et al. Presymptomatic neuropsychological impairment in Huntington's disease. Arch Neurol 1988;45:769-73.

4 Strauss ME, Brandt J. Are there neuropsychological manifestations of the gene for Huntington's disease in asymptomatic, at-risk individuals? Arch Neurol 1990;47: 905-8.

5 Diamond R, White RF, Myers RH, et al. Evidence of presymptomatic cognitive decline in Huntington's disease. presymptomatic cognitive decline in Hun

6 Foroud T, Siemers E, Kleindorfer D, et al. Cognitive scores in carriers of Huntington's disease gene compared to non in carriers of Huntington's disease gene

7 Giordani B, Berent S, Boivin MJ, et al. Longitudinal neuropsychological and genetic linkage analysis of persons at risk for Huntington's disease. Arch Neurol 1995;52:5964.

8 Blackmore L, Simpson A, Crawford JR. Cognitive performance in UK sample of presymptomatic people carrying the gene for Huntington's disease. F Med Genet 1995;32:35862.

9 Broholm J, Cassiman JJ, Craufurd D, et al. Guidelines for the molecular genetics predictive test in Huntington's disease. Neurology 1994;44:1533-6.

10 Dodé C, Dürr A, Pêcheux CV, et al. Huntington's disease in French families: CAG repeat expansion and linkage disequilibrum analysis. C R Acad Sci 1993;316:1374-80.

11 Caine ED, Hunt RD, Weingartner H, et al. Huntington's dementia: clinical and neuropsychological features. Arch dementia: clinical and neurops
Gen Psychiatry 1978;35:378-84.

12 Butters N, Sax D, Montgomery K, et al. Comparison of the neuropsychological deficits associated with early and
advanced Huntington's disease. Arch Neurol 1978;35:585a

13 Brandt J. Cognitive impairments in Huntington's disease: insights into the neuropsychology of the striatum. In: Boller F, Grafman J, eds. Handbook of neuropsychology. New York: Elsevier, 1991:241-64.

14 Pillon B, Dubois B, Ploska A, et al. Severity of cognitive impairment in Alzheimer's, Huntington's, and Parkinson's diseases and progressive supranuclear palsy. Neurology 1991;41:634-43.

15 Folstein MF, Folstein SE, McHugh PR. Mini-mental state. f Psychiatr Res 1975;12:189-98.

16 Mattis S. Dementia rating scale. Odessa: Psychological Assessment Resources, 1988.

17 Wechsler D. Wechsler adult intelligence scale revised. New York: Psychological Corporation, 1981.

18 Stroop JR. Studies of interference in serial verbal reactions. f Exp Psychol 1935;18:643-662.

19 Dürr A, Dodé C, Hahn V, et al. Diagnosis of " sporadic “ Huntington's disease. I Neurol Sci 1995;129;51-5.

20 Cardebat D, Doyon B, Puel M, et al. Evocation lexicale formelle et sémantique chez des sujets normaux. Performances et dynamiques de production en fonction du sexe, de l'âge et du niveau culturel. Acta Neurol Belg 1990;90:20717.

21 Reitan RM. Validity of the trail making test as an indication of organic brain damage. Percept Mot Skills 1958;8:271-6.

22 Wechsler D. Wechlser memory scale. New York: Psychological Corporation, 1945.

23 Delis DC, Kramer JK, Kaplan E, et al. The California verbal learning test, research ed. New York: Psychological Corporation, 1987.

24 Underwood BJ. The role of association in recognition memory. Fournal of Experimental Psychological Monographs 1974;102:917-39.

25 Montgomery SA, Asberg M. A new depression scale designed to be sensitive to change. $\mathrm{Br} f$ Psychiatry 1979;134:382-9.

26 Covi L, Lipman RS. Primary depression or primary anxiety? A possible psychometric approach to a diagnostic dilemma. Clin Neuropharmacol 1984;7(suppl 1):924-5.

27 Strauss ME, Brandt J. Is there increased WAIS pattern variability in Huntington's disease? f Clin Exp Neuropsychol 1985;7:122-6.

28 Rothlind JC, Brandt J, Zee D, et al. Unimpaired verbal memory and oculomotor control in asymptomatic adults with the genetic marker for Huntington's disease. Arch Neurol 1993;50:799-802

29 Josiassen RC, Curry LM, Mancall EL. Development of neuropsychological deficits in Huntington's disease. Arch Neurol 1983;40:791-6.

30 Lezak MD. Neuropsychological assessment, 3rd ed. New York: Oxford University Press, 1995

31 El-Awar M, Becker JT, Hammond KM, et al. Learning deficit in Parkinson's disease. Arch Neurol 1987;44:180-4.

32 Josiassen RC, Curry LM, Roemer RA, et al. Patterns of intellectual deficit in Huntington's disease. 7 Clin Neuropsychol 1982;4:173-83.

33 Brandt J, Strauss ME, Larus J, et al. Clinical correlates of dementia and disability in Huntington's disease. $\mathcal{f}$ Clin Neuropsychol 1984;6:401-12.

34 Smith S, Butters N, White R, et al. Priming semantic relations in patients with Huntington's disease. Brain Lang 1988;33:27-40.

35 Lange KW, Sahakian BJ, Quinn NP, et al. Comparison of executive and visuospatial memory function in Huntington's disease and dementia of Alzheimer type matched for degree of dementia. F Neurol Neurosurg Psychiatry 1995;58: 598-606.

36 Lawrence AD, Sahakian BJ, Hodges JR, et al. Executive and mnemonic functions in early Huntington's disease. Brain 1996;119:1633-45.

37 Massman PJ, Delis DC, Butters N, et al. Are all subcortical dementias alike? Verbal learning and memory in Parkinson's and Huntington's disease patients. I Clin Exp Neuropsychol 1990;12:729-744.

38 Butters N, Granholm E, Salmon DP, et al. Episodic and semantic memory: a comparison of amnesic and demented patients. F Clin Exp Neuropsychol 1987;9:479-97.

39 Pillon B, Deweer B, Agid Y, et al. Explicit memory in Alzheimer's, Parkinson's, and Huntington's diseases. Arch Neurol 1993;50:374-9.

40 Kuhl DE, Phelps ME, Markham CD, et al. Cerebral metabolism and atrophy in Huntington's disease as determined by $18 \mathrm{FDG}$ and computed tomographic scan. Ann Neurol 1982;12:425-34.

41 Kuhl DE, Metter EJ, Riege WH, et al. Patterns of cerebral glucose utilization in Parkinson's disease and Huntington's disease. Ann Neurol 1984;15(suppl):S119-25.

42 Maziotta JC, Phelps ME, Pahl JJ, et al. Reduced cerebral metabolism in asymptomatic subjects at risk for Huntington's disease. N Engl f Med 1987;316:357-62. 